\title{
¿Doctor, dónde está mi abrigo? Intoxicación por aluminio en Hemodiálisis.
}
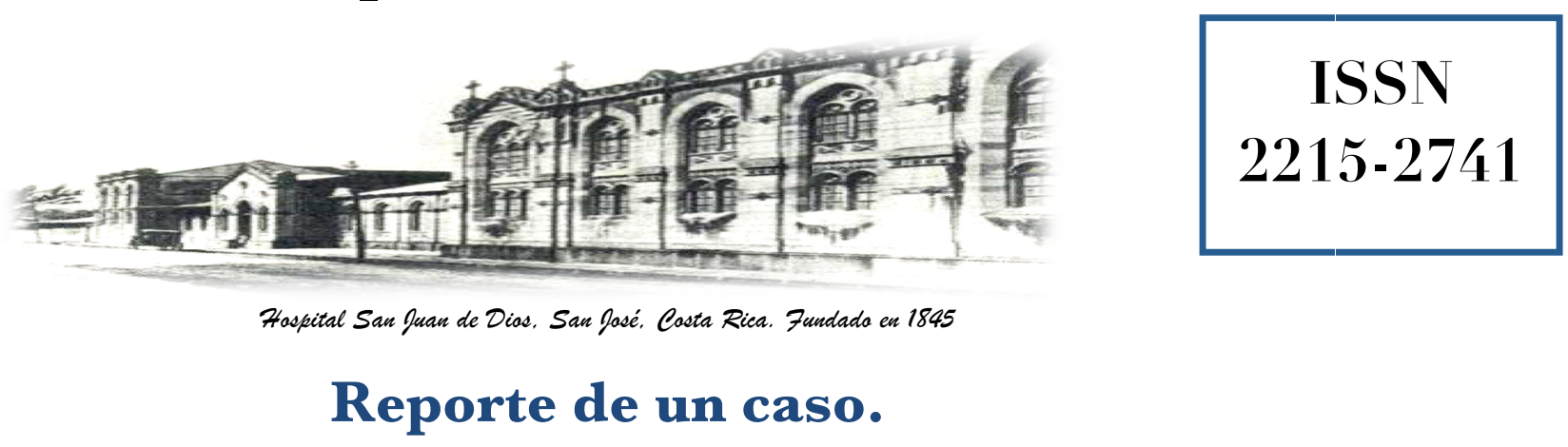

Haspital San quan de Dias. San Dasé. Casta Rica. Fundada en 1845

\section{Reporte de un caso.}

$\begin{array}{ll}\text { Recibido: } & 22 / 09 / 2014 \\ \text { Aceptado: } & 26 / 11 / 2014\end{array}$

\footnotetext{
${ }^{1}$ Médico Especialista en Medicina Interna y Nefrología. Asistente del Servicio de Nefrología - Hospital Dr. Rafael Ángel Calderón Guardia. C.C.S.S.
}

\section{INTRODUCCIÓN}

Se quiere compartir la historia de un paciente masculino de 52 años que desarrolló enfermedad renal crónica a causa de una glomerulonefritis crónica no determinada. Para el año 2004 se le realizó trasplante renal de donador cadavérico. El paciente tenía un buen apego al tratamiento, sin embargo evolucionó con un deterioro progresivo de la función renal del injerto después de los 8 años de su trasplante, por lo que se consideró que fue producto de toxicidad por el inhibidor de calcineurina, del régimen inmunosupresor que utilizaba.

Cuando el paciente se encontraba en clasificación $4 \mathrm{~T}$ de enfermedad renal crónica, se le detectó una masa renal de riñón nativo derecho por lo que fue llevado a nefrectomía total, dando una descripción histopatológica de carcinoma de células claras sin compromiso extracapsular.

El paciente posteriormente se complicó con sepsis severa por infección de la herida quirúrgica y una bronconeumonía nosocomial que lo llevó a lesión renal aguda, con la necesidad de iniciar hemodiálisis de urgencia. Posteriormente a esta crisis, el paciente perdió la reserva renal residual del injerto e ingresó al programa de Hemodiálisis crónica.

Durante los primeros 3 meses de hemodiálisis se logró retirar la inmunosupresión residual a base de esteroides que tenía el paciente y se inició el periodo de observación y espera de no recidiva del tumor previo, para poder programar un segundo trasplante renal.

Inició con deterioro progresivo de su condición nutricional, que ameritó nutrición parenteral intradialítica y concomitantemente desarrolló una hernia incisional gigante que no pudo ser corregida por su desnutrición severa.

En los controles seriados de los exámenes de hemodiálisis se detectó un nivel de hormona paratiroidea $(\mathrm{PTH})$ consistentemente menor de $100 \mathrm{pg} / \mathrm{ml}$ (20 a $35 \mathrm{pg} / \mathrm{ml})$, calcemias de 10.5 a 11 
$\mathrm{mg} / \mathrm{dl}$ sin uso de aporte suplementario de calcio o análogos de vitamina $\mathrm{D}$ y niveles séricos de fosfato entre 6.5 y $7 \mathrm{mg} / \mathrm{dl}$, creatinina sérica de $6.9 \mathrm{mg} / \mathrm{dl}$, BUN: $39 \mathrm{mg} / \mathrm{dl}$ y albúmina: $2.6 \mathrm{~g} / \mathrm{dl}$. De igual manera, hemoglobina de 9 a $10 \mathrm{~g} / \mathrm{dl}$ con VCM:78 fl y HCM: 25 pg, índice de saturación de transferrina: $17 \%$, ferritina: 618 a pesar de todas las medidas correctivas de suplementación de hierro y aumento de eritropoyetina, entre otros factores.

El paciente inició con dolores osteoarticulares severos y mayor debilidad. Debido a la evolución tórpida, se le realizó nuevos estudios para descartar reaparición de la neoplasia, sin embargo, no se logró detectar ninguna lesión. Fue necesario trabajar en conjunto con la Clínica del Dolor para tratar de controlar las molestias osteomusculares.

Un día de rutina de hemodiálsis, posterior a la sesión de diálisis, el paciente se dirige a mi diciendo: “'Doctor! ¿Dónde está mi abrigo?”, le explico que lo tiene puesto y él sonríe. En las próximas sesiones de hemodiálisis vuelve a repetir el mismo patrón, preguntando por su abrigo, billetera y teléfono celular y los familiares alegaron que lo notaban desorientado en el hogar.

Por este motivo se hizo una solicitud de valoración por neurología y se coordinó una tomografía computarizada de cerebro; se concluyó que el paciente cursaba con una encefalopatía metabólica.

Debido a que el paciente llevaba 14 meses del ingreso a hemodiálisis, con consumo diario de 30 $\mathrm{ml}$ de hidróxido de aluminio en cada comida, se decidió enviar una muestra de sangre para análisis de cuantificación de niveles de aluminio con técnica de Absorción Atómica AA800 por Horno de Grafito de la marca Perkin Elmer. Este examen se lleva a cabo en el Laboratorio de Nefrología del Hospital San Juan de Dios y se logró detectar valores de más de $40 \mu \mathrm{g} / 1$.

Con este resultado se planteó que el paciente cursaba con una intoxicación crónica por aluminio. Se intentó iniciar medidas correctivas retirando el hidróxido de aluminio pero sin posibilidad de recibir ningún otro ligador de fósforo. No se logró realizar una biopsia de hueso para corroborar el diagnóstico por la dificultad técnica y de análisis que involucra este procedimiento. Progresó con su deterioro y no se logró administrar desferoxamina.
Lamentablemente falleció y dado que murió en su hogar, no se le realizó autopsia.

\section{DISCUSIÓN}

\section{Situación de la Hemodiálisis en Costa Rica}

Costa Rica es un país de $51.100 \mathrm{~km}^{2}$ con una población aproximada de 4.5 millones de habitantes. Cuenta con un Sistema de Seguridad Social con una red de distribución de servicios de salud dividido en niveles de atención: primaria, secundaria y terciaria. En el nivel terciario de atención (mayor complejidad) hay cuatro hospitales de adultos que cuentan con Servicio de Hemodiálisis, los cuales se encuentran saturados y se manejan de forma estandarizada, sin poder dar una hemodiálisis a la carta para cada paciente.

Dentro del equipamiento se cuenta con máquinas de hemodiálisis con ósmosis inversa y los análisis de agua que se han realizado en diferentes momentos, no han demostrado niveles elevados de aluminio, sin embargo, esta práctica de medición de metales en el agua no es rutinaria ni estandarizada y además, ninguna de las unidades cuenta con un sistema central de purificación del agua, por lo que la calidad del agua de diálisis queda dependiente al funcionamiento de cada una de las máquinas y las condiciones de pureza del agua pueden estar cambiando en forma dramática sin estar percibiéndose, por la ausencia de los análisis correspondientes y periódicos.

\section{Situación de quelantes de fósforo en Costa Rica.}

Con relación a la disposición de quelantes de fósforo, la seguridad social únicamente cuenta con hidróxido de aluminio y se ha dado una lucha de convencimiento a las autoridades competentes para intentar cambiar esta situación, sin embargo, no es hasta principios del 2014 que se muestra una apertura a la adquisición de nuevos quelantes de fósforo, pero con una restricción en su utilización debido a los altos costos, lo que lleva a que no todos los pacientes puedan recibir el quelante sin aluminio y queda sujeto al cumplimiento del protocolo institucional. Por esta razón, nuestro paciente y probablemente muchos más, cursan con niveles elevados de aluminio y las complicaciones que esto conlleva a nivel del sistema nervioso, 
metabolismo mineral óseo, médula ósea y otros.

Definitivamente, los pacientes en diálisis tienen alto riesgo de cursar con sobrecarga de aluminio debido al uso crónico de quelantes de fósforo, agua para diálisis con aluminio y la pobre excreción renal del aluminio ${ }^{(1-3)}$.

Los lineamientos internacionales recomiendan que el líquido de diálisis mantenga niveles de aluminio menores a $10 \mu \mathrm{g} / \mathrm{l}^{(4)}$. En unidades de diálisis con ósmosis inversa se esperaría que se mantengan esos parámetros recomendados, lo que llevaría a los ligadores de fósforo con aluminio, según Savory y Slatopolsky, a ser el principal aporte de exógeno de este metal en los pacientes en diálisis ${ }^{(5,6)}$.

El uso de ligadores de fósforo con aluminio se recomienda por tiempo corto, no más de 8 semanas $^{(7)}$ y este tipo de medicamentos son prohibidos en algunos países ${ }^{(8)}$.

Un estudio retrospectivo de más de 43.000 pacientes en diálisis evidenció que el $2.5 \%$ de los pacientes tuvieron niveles mayores de $50 \mu \mathrm{g} / 1 \mathrm{y}$ disminuyeron significativamente cada año ${ }^{(9)}$. Sin embargo, la prevalencia de sobrecarga de aluminio podría ser más elevada en países que comúnmente utilizan ligadores de fósforo que contienen aluminio $^{(10)}$ y ésta es la situación en nuestra región.

\section{Manifestación de la intoxicación por Aluminio en relación al paciente.}

El aluminio es un metal que puede acumularse en diferentes órganos y sistemas del cuerpo, lo que puede llevar al desarrollo de varias enfermedades, incluyendo la encefalopatía asociada a diálisis, la enfermedad ósea inducida por aluminio, la hipercalcemia y la anemia resistente al hierro $^{(7,11,12)}$.

Nuestro paciente cursó con encefalopatía, anemia resistente a los suplementos farmacológicos, hipercalcemia, PTH baja y dolores osteomusculares. Todo este cuadro clínico con un nivel elevado de aluminio concomitante hace pensar en definitiva, que tuvo una intoxicación crónica con este metal.

Con relación a la encefalopatía por aluminio, se describe que generalmente es de una progresión lenta y los síntomas aparecen alrededor de un año de estar en hemodiálisis y se caracterizan por cambios mentales, alteraciones del lenguaje, alucinaciones visuales o auditivas, comportamiento paranoide y hasta convulsiones. Todos estos signos y síntomas pueden fluctuar y pueden empeorar después de la sesión de hemodiálisis ${ }^{(13)}$.

La neurotoxicidad del aluminio seguida de la exposición oral ha sido bien establecida en humanos con insuficiencia renal, por lo que esta población de pacientes tiene riesgo de desarrollar demencia relacionada al aluminio ${ }^{(14)}$. Una prolongada exposición al aluminio en el líquido de diálisis o combinado con la ingesta oral del hidróxido de aluminio puede producir el llamado síndrome de demencia de la diálisis, descrito por Alfrey, en donde ordena al síndrome en dos tipos de neurotoxicidad: Agudo y clásico ${ }^{(15)}$.

Agudo: esta forma de presentación del síndrome es causada por altos niveles de aluminio, por la ingestión de ligadores de fósforo con aluminio y citrato o por la rápida liberación sérica seguida del tratamiento con desferoxamina. El inicio de la neurotoxicidad es rápido, con una marcada confusión y espasmos musculares; además puede desarrollar convulsiones, coma y muerte. Los niveles de aluminio en plasma típicamente son mayores de $500 \mu \mathrm{g} / \mathrm{l}$, tomando en cuenta que los niveles normales son de 1 a $3 \mu \mathrm{g} / \mathrm{l}^{(16)}$.

Clásico: este tipo resulta de la exposición parenteral u oral al aluminio y se caracteriza por un inicio gradual, con desórdenes del comportamiento y eventualmente la muerte. Los efectos neurológicos se han observado tanto en niños como en adultos ${ }^{(14)}$. Los niveles séricos son estimados entre 100 y $200 \mu \mathrm{g} / 1$.

Limitar la exposición al aluminio en pacientes urémicos con el uso de ligadores de fósforo libres de aluminio eliminaría el riesgo de esos efectos neurotóxicos ${ }^{(17)}$.

La anemia asociada con la sobrecarga de aluminio se relaciona a varios mecanismos que afectan al proceso normal de la hematopoyesis. Se ha descrito alteración de la absorción intestinal, el transporte sérico y la captación celular del hierro debido a que el hierro y al aluminio comparten las vías de absorción y son transportados por las mismas proteínas, como por ejemplo transferrina 
y la albúmina entre otras ${ }^{(18)}$. Por otra parte, se ha descrito un efecto de resistencia hematopoyética a la eritropoyetina recombinante, humana inducida por el aluminio ${ }^{(19)}$, esto puede explicar que los pacientes con sobrecarga de aluminio pueden tener altas dosis de eritropoyetina y aumento de los costos de la terapia de diálisis ${ }^{(20)}$.

Sobre el metabolismo mineral óseo se describen varias alteraciones que genera el aluminio, tanto a nivel propiamente de la formación de hueso como sobre el calcio y la hormona paratiroidea (PTH).

A nivel óseo, se describen tres efectos patológicos del aluminio sobre el hueso:

1. Inhibición de la formación de hidroxiapatita

2. Inhibición de la proliferación de células óseas y

3. Inhibición de la actividad de las células óseas.

Estos mecanismos llevan a una mala formación y calidad de hueso $^{(21)}$.

\section{Hipercalcemia}

La enfermedad ósea relacionada al aluminio puede cursar con hipercalcemia ${ }^{(22)}$ y el aluminio genera inhibición de la secreción de la hormona paratiroidea e inhibición de la expresión del ARNm para $\mathrm{PTH}^{(23)}$, esto explicaría la persistencia de PTH baja en nuestro paciente. Por esta razón, en los pacientes con exposición a aluminio que cursen con niveles de PTH bajos e hipercalcemia, debe siempre tenerse en mente que pueden tener sobrecarga de aluminio ${ }^{(13)}$, esto con el fin de tomar las medidas correctivas necesarias.

Los dolores osteomusculares se reportan como comunes en personas expuestas al consumo de aluminio $^{(17)}$. La osteomalacia, caracterizada por un ablandamiento del hueso y aumento de riesgo de fracturas espontáneas, han sido bien documentadas en pacientes urémicos en diálisis, expuestos a ligadores de fósforo que contienen aluminio $^{(24)}$. En relación a esto, el trabajo de Bern demostró que las manifestaciones osteomusculares en pacientes urémicos con exposición a aluminio fueron debilidad muscular en el $27 \%$, dolor óseo en el $18 \%$ y fracturas óseas en $8 \%{ }^{(25)}$.

\section{Tratamiento}

La primera medida a tomar en el tratamiento de sobrecarga de aluminio es la prevención y evitar el uso de agua de diálisis con aluminio y los ligadores de fósforo con aluminio. En el momento que se determine la sobrecarga de aluminio, se recomienda tratar a estos pacientes con desferoxamina, sin embargo, este medicamento tiene efectos secundarios a tomar en cuenta como la neurotoxicidad ${ }^{(26)}$. Sin embargo, la mayoría de esos efectos son leves y reversibles y tienden a ser dosis dependiente, por lo que tienden a ser comunes si las dosis son entre 20 y $40 \mathrm{mg} / \mathrm{Kg}^{(27)}$.

Actualmente, la dosis estándar recomendada es de $5 \mathrm{mg} / \mathrm{kg}^{(7)}$, sin embargo hay estudios que han demostrado que dosis inferiores $(2.5 \mathrm{mg} / \mathrm{kg})$ son efectivas y con bajos efectos secundarios ${ }^{(28)}$.

\section{Apertura institucional a ligadores de fósforo sin aluminio}

La mayoría de nuestros pacientes en diálisis cursan con productos Calcio x Fósforo mayores a 55 , lo que limita utilización exclusiva de ligadores de fósforo con calcio, por lo que se debería contar también con opciones de ligadores de fósforo sin calcio como el carbonato de sevelamer.

En nuestro país se ha dado la lucha con las autoridades competentes demostrando la urgencia de cambiar los ligadores de fósforo con aluminio a otras alternativas como el acetato de calcio y el carbonato de sevelamer. El incremento en los costos ha sido una limitante para dar un cambio rápido y sustituir de una ves por todas el hidróxido de aluminio y esto probablemente es el mismo problema en el resto de la región centroamericana.

La eliminación del uso de aluminio llevaría a menos anemia resistente a eritropoyetina, con la consiguiente disminución de las dosis del análogo de la eritropoyesis, a menos trastornos del metabolismo mineral óseo y las complicaciones derivadas y definitivamente a menos presencia de casos de "encefalopatía de la diálisis" y esto generaría una reducción en los costos de tratamiento en diálisis. Para corroborar esta aseveración, se debería contar con un estudio serio de farmacoeconomía, que contemple todas estas variables.

La otra alternativa es pensar en la compra regional 
a nivel centroamericana de estos fármacos ligadores de fósforo no cálcicos, como el carbonato de sevelamer (renvela ${ }^{\circledR}$ ) ya que muy probablemente el mayor consumo disminuya los precios.

El problema está, nuestros pacientes están sufriendo la exposición crónica al aluminio y hay que detenerlo.

\section{BIBLIOGRAFÍA}

1. Jaffe JA Liftman C Glickman JD. Frequency of elevated serum aluminum levels in adult dialysis patients. Am J Kidney Dis. 2005;46(2):316-319.

2. Humpfner A Hummel S Schultz W. Diagnosis and therapeutic approaches to aluminum overload in dialyzed patients-representative study questionnaire in West German dialysis units in 1989-1990. Nephrol Dial Transplant. 1993;8:S51-S54.

3. Salusky IB. A new era in phosphate binder therapy: What are the options? Kidney Int. 2006;105:S10-S15.

4. Fernández-Martin JL Canteros A Serrano M Gonzalez-Carcedo A Díaz-Corte C Cannata, JB. Prevention of aluminum exposure through dialysis fluids. Analysis of changes in the last 8 years. Nephrol Dial Transplant. 1998;13: S78-S81.

5. Savory J Berlin A Courtoux C Yeoman B Wills M. Summary report of an international workshop on "The role of biological monitoring in the prevention of aluminum toxicity in man: Aluminum analysis in biological fluids." Ann Clin Lab. 1989;13: 444-451.

6. Slatopolsky E. The interaction of parathyroid hormone and aluminum in renal osteodystrophy. Kidney Int. 1987;31(3):842854.

7. National Kidney Foundation. K/DOQI clinical practice guidelines for bone metabolism and disease in chronic kidney disease. Am J Kidney Dis. 2003;42:S108S122.

8. Koiwa F Sato Y. Chemistry and history of phosphate binder. Clin Calcium. 2009;19: 198-204.

9. Jaffe JA Liftman C Glickman JD. Frequency of elevated serum aluminum levels in adult dialysis patients. Am J Kidney Dis. 2005;46
(2):316-319.

10. Kan WC Chien CC Wu CC Su SB Hwang JC Wang HY. Comparison of low-dose deferoxamine versus standard-dose deferoxamine for treatment of aluminium overload among haemodialysis patients. Nephrol Dial Transplant. 2010;25(5):16041608.

11. Berlyne GM Pest D Ben-Ari J et al. Hyperaluminaemia from aluminum resins in renal failure. Lancet. 1970;296(7671):494496.

12. Sherrard DJ. Letter: the myth of aluminum toxicity. N Engl J Med. 1974;290:750.

13. Wei-Chih K Chih-Chiang Ch Yi-Hua L JyhChang H Shih-Bin S Hsien-Yi W. Aluminum Overload: An Easily - Ignored Problem in Dialysis Patients with Hyperparathyroidism. ISBN: 978-953-51-0478-0, InTech, Available from:

http://www.intechopen.com/books/hyperparat hyroidism/aluminum-overload-an-easilyignored-problem-in-dialysis-patients-withhyperparathyroidism

14. Alfrey AC. Aluminum toxicity in patients with chronic renal failure. Ther Drug Monit. 1993;15(6):593-597.

15. Alfrey AC LeGendre GR Kaehny WD. The dialysis encephalopathy syndrome: Possible aluminum intoxication. $\mathrm{N}$ Engl J Med. 1976;294(4):184-188.

16. House RA. Factors affecting plasma aluminum concentrations in nonexposed workers. J Occup Med. 1992;34:1013-1017.

17. U.S. department of Health and Human Services. Public Health Service Agency for Toxic Substances and Disease Registry. Toxicological profile for aluminum. September 2008.

18. Cannata JB Fernández-Soto I FernándezMenéndez MJ et al. Role of iron metabolism in absorption and cellular uptake of aluminum. Kidney Int.1991;39(4):799-803.

19. Losekann A Ureña $\mathrm{P}$ Khiraoui $\mathrm{F}$ et al. Aluminium intoxication in the rat induces partial resistan e to the effect of recombinant human erythropoietin. Nephrol Dial Transplant. 1990;5(4):258-263.

20. Drüeke TB. Resistance to recombinant human erythropoietin in hemodialysis patients. Am J Nephrol. 1990;10(2):S34-S39.

21. Malluche H. Aluminum and bone disease in chronic renal failure. Nephrol Dial Trasplant. 2002;17(2):S21-S24. 
22. Norris KC Crooks PW Nebeker HG et al. (1985). Clinical and laboratory features of aluminum-related bone disease: Differences between sporadic and "epidemic" forms of the syndrome. Am J Kidney Dis. 1985;6:342347.

23. Diaz-Corte C Fernandez Martin JL. Effect of aluminium load on parathyroid hormone synthesis. Nephrol Dial Trasplant. 2001;16: 742-745.

24. Andreoli SP Bergstein JM Sherrard DJ. Aluminum intoxication from aluminumcontaining phosphate binders in children with azotemia not undergoing dialysis. N Engl J Med. 1984;310:1079-1084.

25. Brem AS DiMario C Levy DL. Perceived Aluminum-Related Disease in a Dialysis Population A Report From the End-Stage Renal Disease Network 28. Arch Intern Med. 1989;149:2541-2544.

26. McCauley J Sorkin MI. Exacerbation of aluminum encephalopathy after treatment with desferrioxamine. Nephrol Dial Transplant. 1989;4(2):110-114.

27. Bene C Manzler A Bene D Kranias G. Irreversible ocular toxicity from single "challenge" dose of deferoxamine. Clin Nephrol. 1989;31(1):45-48.

28. Kan WC Chien CC Wu CC Su SB Hwang JC Wang HY. Comparison of low-dose deferoxamine versus standard-dose deferoxamine for treatment of aluminium overload among haemodialysis patients. Nephrol Dial Transplant. 2010;25(5):16041608 . 